Article

\title{
Polyamide 11/Poly(vinylidene fluoride)/Vinyl Acetate-Maleic Anhydride Copolymer as Novel Blends Flexible Materials for Capacitors
}

\section{Dongliang Kuang ${ }^{1}$, Rui $\mathrm{Li}^{2}$ and Jianzhong Pei ${ }^{2, *}$}

1 School of Materials Science and Engineering, Chang'an University, Xi' an 710064, China; E-Mail: kuangdl@163.com

2 Highway School, Chang’an University, Xi’an 710064, China; E-Mail: lirui@chd.edu.cn

* Author to whom correspondence should be addressed; E-Mail: peijianzhong@126.com; Tel./Fax: +86-29-6263-0058.

Received: 2 January 2014; in revised form: 28 March 2014 / Accepted: 2 April 2014 /

Published: 11 August 2014

\begin{abstract}
A novel all-polymeric blend with high dielectric constant $(\mathrm{K})$ has been developed by blending polyvinylidene fluoride (PVDF) and polyamide (PA11) via co-melt-pressing technology with a compatilizer vinyl acetate-maleic anhydride (VA-MA) copolymer. Adding a copolymer of vinyl acetate and maleic anhydride decreased the dielectric loss ( $\tan \delta \approx 0.057)$ and increased the dielectric constant $\left(K_{\text {blend }}=15\right)$. The blends show high dielectric constants, which give better frequency stability, and excellent mechanical properties. SEM investigations suggest that the enhanced dielectric behavior originates from significant interfacial polymer-polymer interactions. FT-IR, DSC and XRD demonstrate that blending PA11 with PVDF affects the crystalline behavior of each component. The high-K polymeric blends created represent a novel type of material that is flexible and easy to process, moreover, is appropriate for flexible micro-electronics.
\end{abstract}

Keywords: all-polymeric blend; dielectric property; VA-MA; intermolecular interaction

\section{Introduction}

Polyvinylidene fluoride (PVDF) and its polymeric composites, as the typical polymers of electric properties [1-4], have been widely studied in piezoelectricity and dielectric property since the discovery of their electric properties [5,6]. In early reports, inorganic-polymer composites such as Piezoelectric 
lead zirconate titanate (PZT)/PVDF composite have been developed, and much attention has been paid to the dielectric properties studies [7-10]. High content of ceramic powders (usually over 50 vol\%) in the polymer composites can enhance the dielectric constant but also makes the polymer matrix lose its flexibility and deteriorates the adaptability [11-14], the mechanical properties, machine-shaping process resulted from the inorganic component has slowed the progress in the application field.

It is generally known that a macromolecule posses unique properties due to their architectural feature. The polymer-polymer blends are now gaining considerable research attention for applications in inductive and capacitive components sectors, because they can provide light weight, flexible, and volume efficient electrical components required for embedded passive technologies and others.

On the other hand, the blending of different polymers is an important strategy to obtain the desired properties of polymer systems, which is based on the structure and property of each component [15-17]. In the review by Scheinbeim et al. $[18,19]$, the authors reported the odd-numbered Nylons such as polyamide (PA11) exhibiting fine piezoelectric behavior by appropriate electro processing, especially when the temperature is raised above the glass transition temperature of the nylons [20,21]. However, most polymer blends are immiscible and form a multiphase structure because of the high molecular weight and unfavorable interactions between the component polymers. Typically, phase separation exhibited by two polymers occurs on a size scale of several microns, which gives rise to opacity and can cause a deterioration of properties due to poor interfacial adhesion. In the field of polymer blends, interfacial interactions play a critical role in determining material dielectric properties. In our previous study, we reported the PA11/PVDF blend displayed a high dielectric constant [22], but the preparation process is complex with low yield and length time, which is difficult for application.

In this paper, we put forward a simple and effective route to prepare high dielectric behavior polymeric blends with mechanical properties and flexible property for a capacitor. Meanwhile, the alternating copolymer from vinyl acetate and maleic anhydride was employed as an excellent compatilizer for the PA11/PVDF blends, through analyzing the electric properties from the structural mechanism so as to gain a better electric properties material. The goal of our research is to make polymer blends with high dielectric constant in an easy way, without the addition of any inorganic additives.

\section{Experimental Section}

\subsection{Materials}

$\operatorname{PVDF}\left(M_{\mathrm{W}}=36 \times 10^{5}, d=1.75 \mathrm{~g} / \mathrm{cm}^{3}\right)$ and PA11 $\left(M_{\mathrm{W}}=4 \times 10^{5}, d=1.03 \mathrm{~g} / \mathrm{cm}^{3}\right)$ powders were supplied by the Shanghai 3F Corporation and the Guangzhou Far East Chemistry Corporation, respectively. Vinyl acetate, maleic anhydride, toluene and azodiisobutyronitrile (AIBN) were purchased from Shanghai Chemical Reagent Company and used as received.

\subsection{Methods}

\subsubsection{Synthesis of Copolymer of Vinyl Acetate and Maleic Anhydride}

The copolymer was prepared according to the following reactions (Scheme 1). Firstly, maleic anhydride $(0.09 \mathrm{~mol})$ was dissolved in toluene and heated until the solution complete clarification with 
vigorous stirring. After this, vinyl acetate $(0.09 \mathrm{~mol})$ and AIBN $(0.0012 \mathrm{~mol})$ were added to the toluene solution. The reaction was allowed to continue for $2 \mathrm{~h}$ under stirring at about $85{ }^{\circ} \mathrm{C}$. The resulting copolymer was filtered and washed with toluene for several times, then dried at $100{ }^{\circ} \mathrm{C}$ for $12 \mathrm{~h}$ under vacuum to afford a white powder.

Scheme 1. Synthesis of vinyl acetate-maleic anhydride (VA-MA) alternating copolymer.<smiles>C=COC(C)=O</smiles>

\subsubsection{Composition of PA11/PVDF and PA11/VA-MA/PVDF}

The PA11/PVDF and PA11/VA-MA/PVDF blends, $12 \mathrm{~mm}$ in diameter and $1.2 \mathrm{~mm}$ in thickness, were prepared from PA11, VA-MA and PVDF powders by following a simple blending and hot-pressing procedure at about $180^{\circ} \mathrm{C}$ and $20 \mathrm{MPa}$ for $30 \mathrm{~min}$. Finally, the samples were polished and silvered by silver-paint.

\subsection{Instrumental Analysis}

The samples were left at room temperature for $24 \mathrm{~h}$, and then the dielectric properties were measured by HIOKI3532-50 LCR. The electrically polarization is along the thickness direction at $2 \sim 4 \mathrm{kV}$ for $25 \mathrm{~min}$ at room temperature. After being polarized, the samples were left at room temperature for $24 \mathrm{~h}$, and the piezoelectric properties were then measured on a ZJ-3A $\mathrm{d}_{33}$ test machine. Fourier Transform Infrared Spectroscopy (FT-IR) of PA11/PVDF blends were measured by a Nicolet FTIR infrared rays spectrum. In order to analyze the compatibility and flexibility of the blends, the surface topographies of PA11/PVDF blends with and without VA-MA were observed by a JSM-5610LV scanning electron microscope (SEM).

\section{Results and Discussions}

\subsection{Dielectric Properties}

A series of PA11/VA-MA/PVDF and PA11/PVDF blends with various volume fractions of PVDF and VAMA were prepared by using a very simple hot-pressing procedure.

Figure 1a shows the frequency dependence of the dielectric constant of the PA11/PVDF blends. The dielectric constant increases with the number of PVDF used in the hot press initially, but it is still very low. The dielectric constant $\left(\mathrm{K}_{\text {blend }}\right)$ of PA11/PVDF blends located between 7 and 10, observed distinctly for the film at room temperature. We also know that the PVDF in the system play a major role in the electrical properties. Because VA-MA improves the compatibility between PA11 and PVDF, the dielectric constants of PA11/PVDF blends containing VA-MA increase remarkably (Figure 1b). For example, when the ratio of PA11 to PVDF is 20:80, the dielectric constant $\left(K_{\text {blend }}=15.1\right)$ of the blend containing $1 \%$ VA-MA is about three times higher than that of PA11 $(\mathrm{K}=5)$ and nearly two times 
higher than that of PVDF $(K=8)$. Figures $1 \mathrm{c}, \mathrm{d}$ show the frequency dependence of the dielectric loss $\tan \delta$ of the two blends without and with VA-MA, respectively. Comparing Figure 1d with Figure 1c, we demonstrate that, when the ratio of PA11 to PVDF is 20:80, the tan $\delta$ of the blend containing VA-MA $1 \mathrm{wt} \%$ is 0.03 , which is much lower than the value of 0.062 for pure PA11/PVDF blends. It is clearly that the compatibility of PA11 and PVDF affects the dielectric property of the blends. Therefore, the PA11/PVDF blends studied here with a high dielectric constant and a low dielectric loss have high potential as dielectric materials to be employed in the microelectronic package and other industrial applications.

Figure 1. Frequency dependence of dielectric constant for polyamide/polyvinylidene fluoride (PA11/PVDF) blends: (a) without VA-MA; and, (b) with VA-MA added. Frequency dependence of dielectric loss, $\tan \delta$, for PA11/PVDF blends: (c) without VA-MA; and, (d) with VA-MA added.
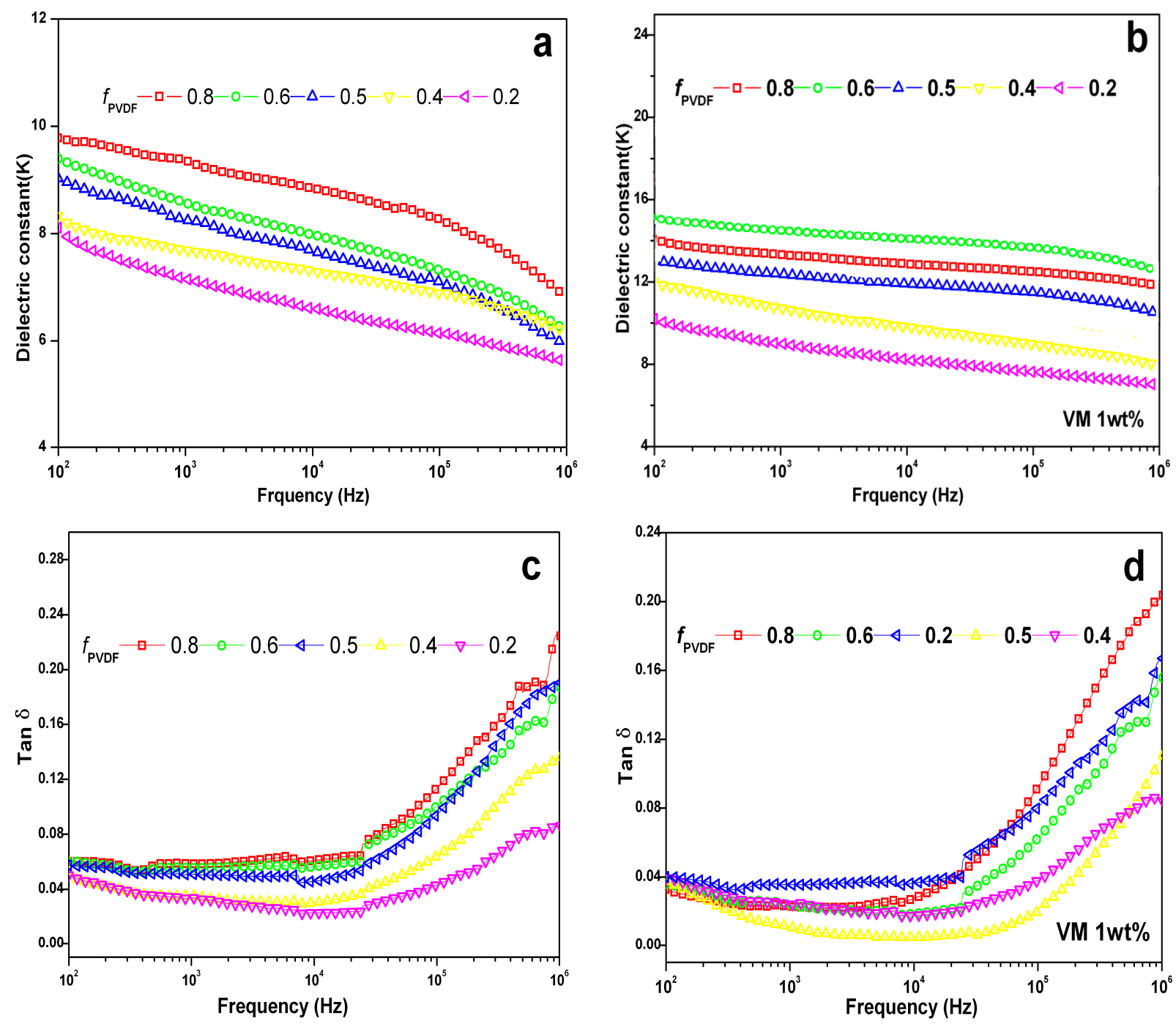

Figure 2 shows the dependence of the dielectric properties of the PA11/PVDF blends on the selected frequency $\left(10^{3} \mathrm{~Hz}\right)$ with different volume fractions of PVDF at room temperature. We can see from Figure 2 clearly that, with increasing PVDF content, the constant peak becomes obvious. This result can be attributed to the contribution of the polar effect of PVDF in the blends. Figure 2 
further confirms the contribution of PVDF. With an increasing volume fraction of PVDF in the blends, the dielectric constant increases on the whole. In this case, when the weight faction of VA-MA reach $5 \mathrm{wt} \%$, the dielectric constant increases rapidly with an increase in the volume fraction of PVDF. When the content of compatilizer increases from $1 \%$ to $5 \%$, the dielectric constant of the system increases largely, and then as the compatilizer content increases to $10 \%$, the dielectric constant of the system began to decrease. However, the dielectric losses of the sample added compatilizer have on the whole done the same as in the pure PA11/PVDF blends: the value of the dielectric losses is less than 0.08, only when the frequency increased to $10^{5} \mathrm{~Hz}$, did the value suddenly increase. This is due to the content of compatilizer being higher than $5 \%$. This effected the crystallization of PVDF, thus limiting the formation of $\beta$-phase PVDF, leading to the content of compatilizer being higher than $5 \%$ and the decline of the dielectric constant of the sample in situation. This is very important for the use of this kind of blend as a dielectric material in capacitors.

Figure 2. Variations of (a) the dielectric constant and (b) the dielectric loss of PA11/PVDF blend versus volume fraction of PA11 at room temperature on $10^{3} \mathrm{HZ}$.

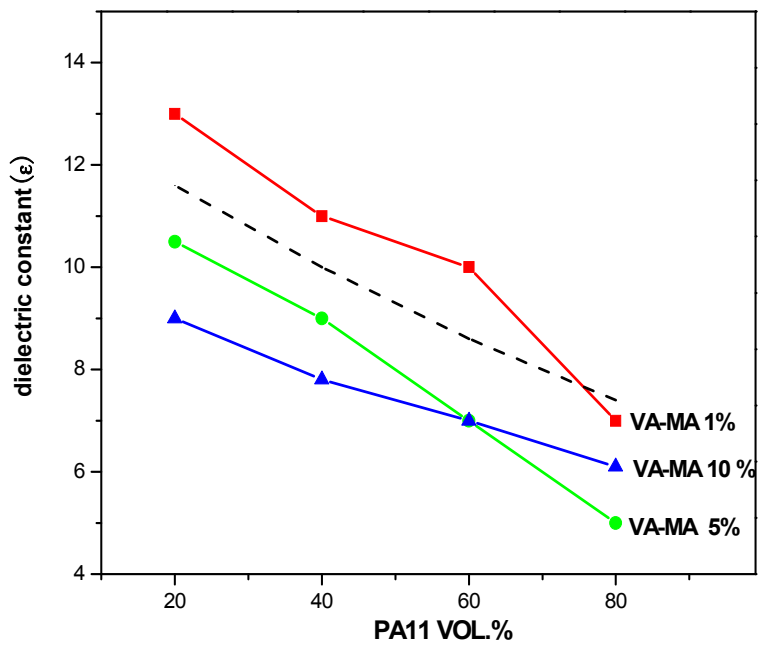

(a)

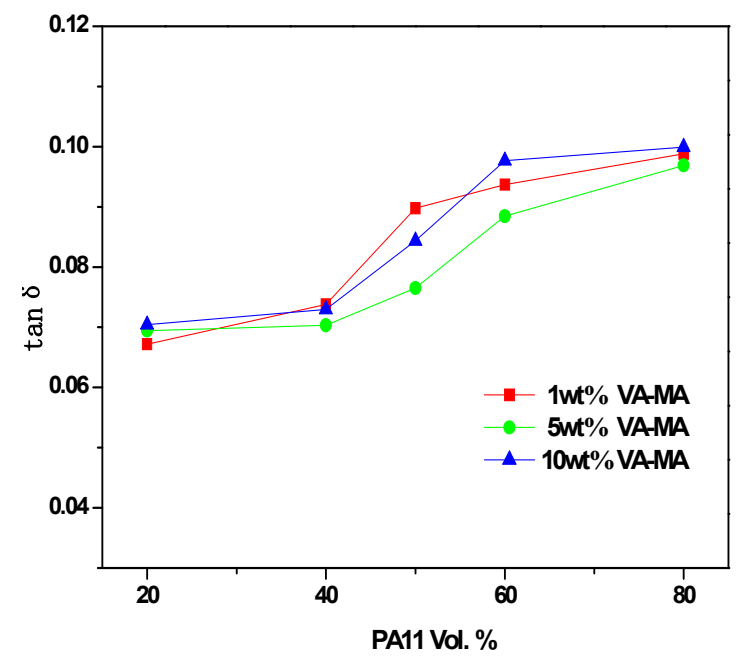

(b)

\subsection{Piezoelectric Properties}

The PA11/PVDF blends with $5 \mathrm{wt} \%$ VA-MA is chosen for studying piezoelectric coefficient $\left(\mathrm{d}_{33}\right)$ owing to the highest dielectric constant and good interfacial compatibility. Figure 3 shows the relationship between the piezoelectric constant $\mathrm{d}_{33}$ and the PVDF volume fraction. Although the two samples do not exhibit super piezoelectric properties, we can see that the blends have better piezoelectricity than PA11 $(2.0 \mathrm{pC} / \mathrm{N})$, and also have a very clear enhancement in the piezoelectric constant $d_{33}$ of the PA11/PVDF by adding VA-MA at room temperature. The results show that, when the content of PVDF is $50 \%$ the polarization degree of PA11/PVDF was enhanced by VA-MA so as to improve the piezoelectric properties. 
Figure 3. The piezoelectric constant $d_{33}$ of PA11/PVDF blends.

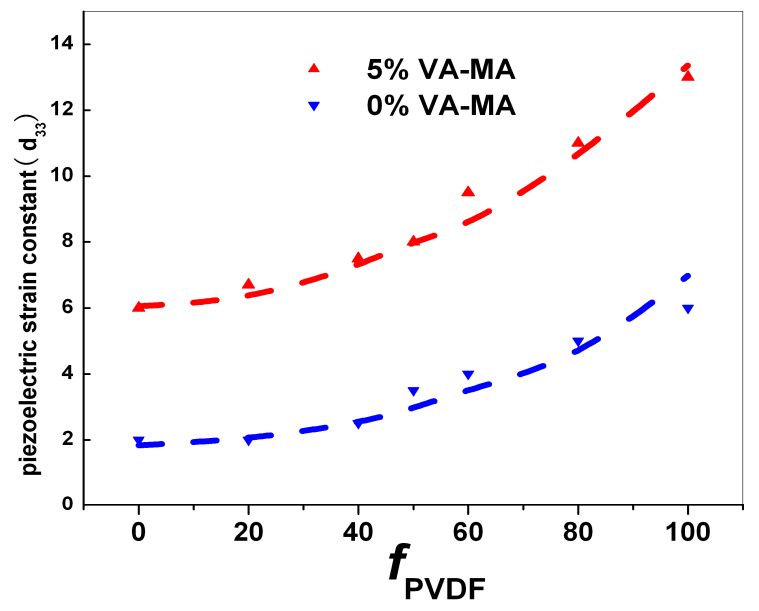

\subsection{Mechanical Properties}

The effect of the PVDF volume fraction on the tensile strength and the tensile elongation of blends are shown in Figure 4. It can be seen that the tensile strength changed irregularly and continually with the increasing content of PVDF. With the increasing content of PVDF, the tensile strength of PA11/PVDF blends decreased sharply, but subsequently increased slowly, and the lowest tensile strength is $16.39 \mathrm{MPa}$ when the PVDF volume was 40\%. Furthermore, the tensile elongation of the PA11/PVDF blends is from $70 \%$ to $20 \%$ and excelled inorganic-polymer composites as dielectric materials. This suggests that the PA11/PVDF blends are a flexible material with high dielectric constant, that is surpassed by only a few other materials.

Figure 4. Variations of the tensile strength and the tensile elongation of PA11/PVDF/VA-MA (5 wt\%) blend versus volume fraction of PVDF.

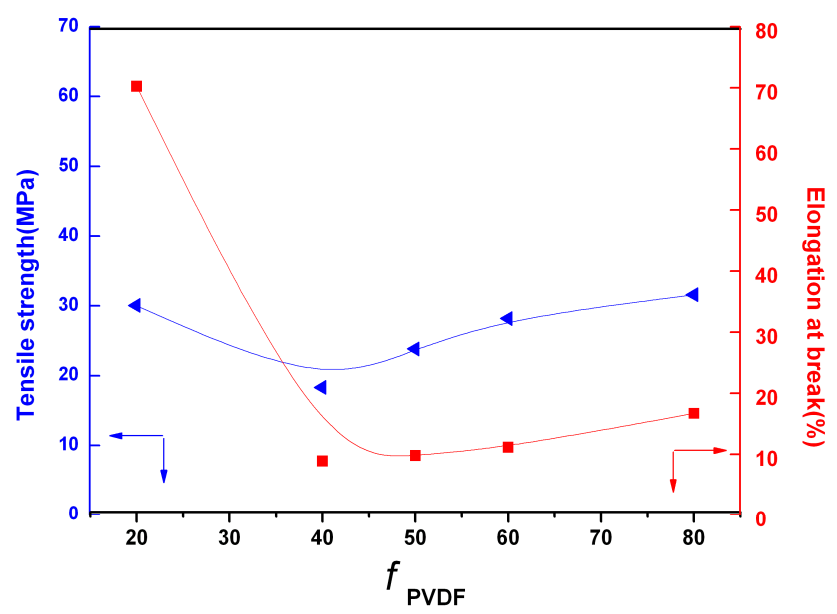

\subsection{Structure Analysis}

The X-ray diffraction (XRD) patterns of PA11/PVDF blends are shown in Figure 5a. We note that the diffraction peak of PA11 appears to strengthen compared to the pure PA11/PVDF. This suggests that the crystallization transmutation of PA11 from $\alpha$ to $\delta^{\prime}$ is served by the compatilizer (VA-MA). Moreover, 
the highly oriented $\alpha$-form PVDF, as already observed in Figure 5a, and those associated with its $\beta$-modification can now clearly be identified. Namely, for the PA11/PVDF blends, the enhanced dielectric properties can be attributed to the VA-MA, which was taken as a "bridge" between the PA11 and PVDF so as to produce electricity. The studies provide additional confirmation that the compatilizer has a greater impact on the interfacial interaction.

Figure 5. (a) The XRD spectra and (b) The DSC for PA11/PVDF (40:60) at $5{ }^{\circ} \mathrm{C} \mathrm{min}^{-1}$.
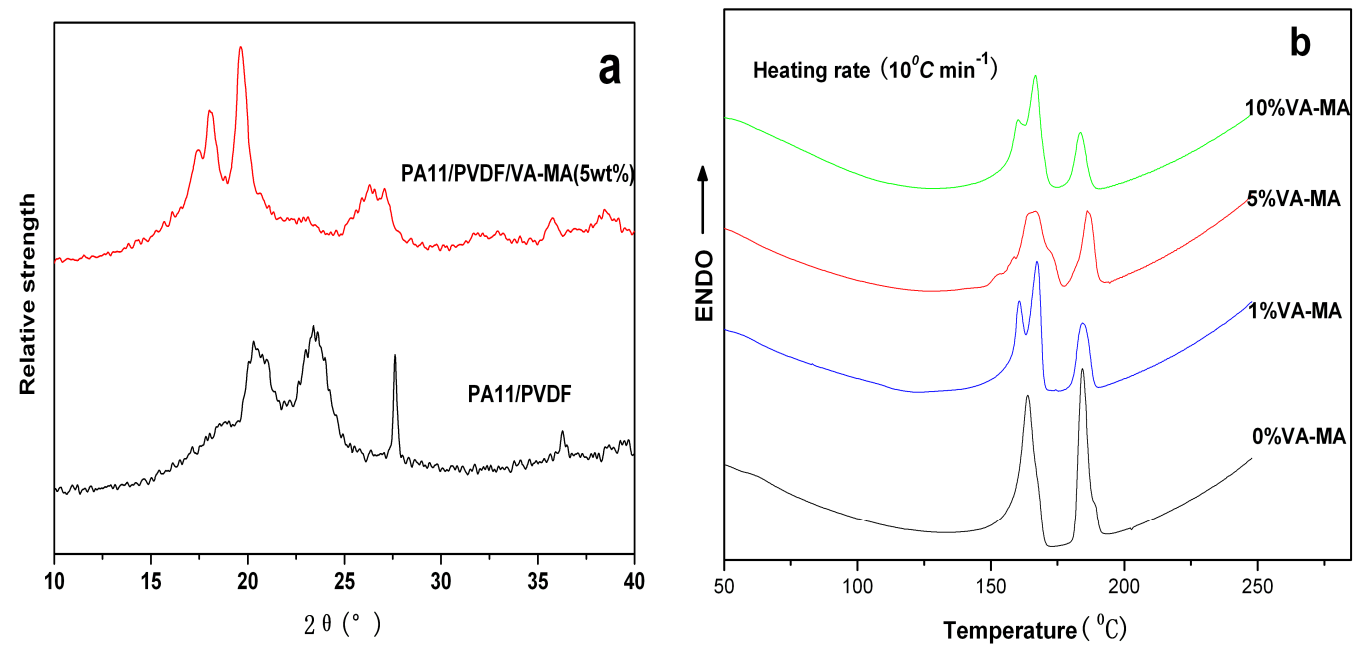

The differential scanning calorimetry (DSC) curves for PA11/PVDF blends with different volume contents of VA-MA are presented in Figure $5 \mathrm{~b}$. We found that the crystallize peaks of the two polymers (PA11 and PVDF) exist in the blends, and the melting zones of the crystals in PA11 and PVDF get broader. The results demonstrate that the crystals of PA11 and PVDF in the blends are incomplete. With the addition of VA-MA, we found that the melting points of two samples got closer and closer and a significant new melting point was seen at about $160{ }^{\circ} \mathrm{C}$. These changes may confirm the existence of specific intermolecular interactions between the PA11 and VA-MA. Also, the VA-MA forms hydrogen bonds with PVDF. More importantly, when the VA-MA is $5 \mathrm{wt} \%$, the compatibility between the two polymers achieves the best situation while the dielectric properties of the PA11/PVDF blend obtain the largest value. The DSC studies provide confirmation that the compatibility can have a greater impact on the dielectric properties. In addition, the PA11/PVDF blends present a melting peak temperature very similar to that of the PVDF matrix because the melting temperatures of PVDF and PA11 are very similar. This is very important for the blending processes used to fabricate PVDF thin films in the future.

As we know, the miscibility played a crucial role in deciding the morphology and properties of the PA11/PVDF blends. Figure 6 is the scanning electron micrograph (SEM) of the PA11/PVDF blend and shows the cross section topography of PA11/PVDF blends after 24-h at room temperature. As shown in Figure 6a, the boundaries between PA11 and PVDF were obvious. It especially exhibited a typical two phase separation, which indicates that PA11 has poor compatibility with PVDF in the blends.

Compared with Figure 6a, Figure 6b exhibits a good compatibility in the blend. A well-connected interface can be clearly observed in virtue of the compatible properties of VA-MA, and there is a remarkable layer-layer interface in the likeness of "a book". This can be ascribed to the interfacial tension between PA11 and PVDF which shows a sharp decrease by the PA11-g-VA-MA. Also, the 
addition of VA-MA made the dispersion more even. That is to say that the presence of VA-MA in blends can improve interface adhesive properties formed as the intermolecular interface.

Figure 6. The SEM of (a) PA11/PVDF and (b) PA11/VA-MA/PVDF.
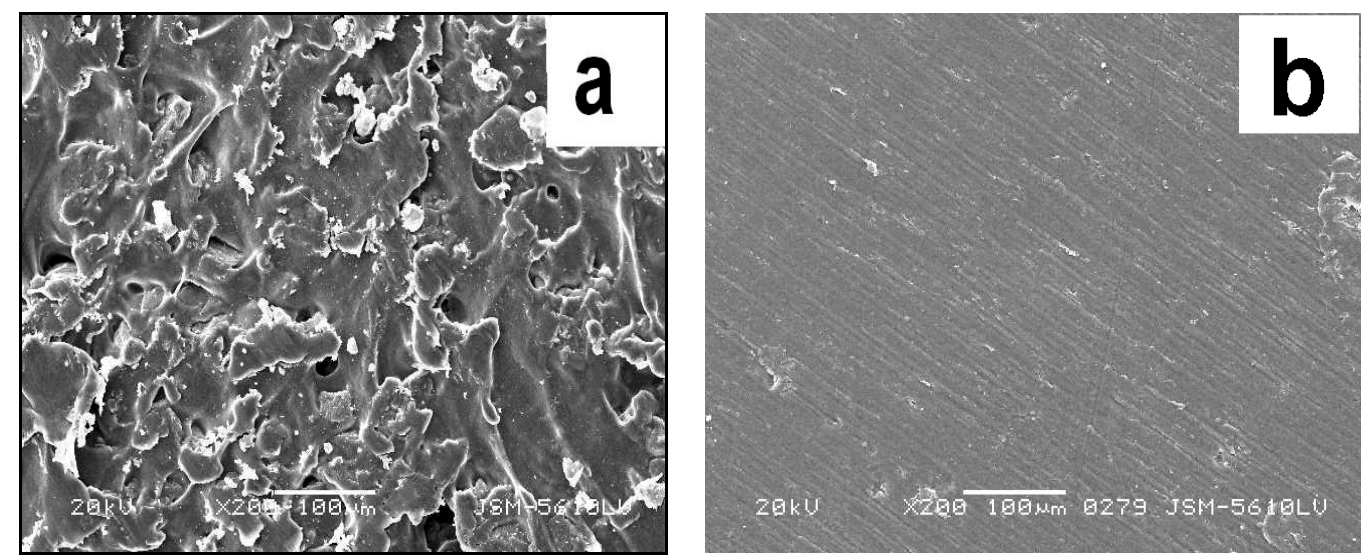

To explore the results for compatilizer properties, we investigated the functionalization of the VA-MA using Fourier transform infrared spectroscopy (FT-IR). Figure 7 is the FT-IR spectrum analyses of the copolymer VA-MA and PA11/VA-MA/PVDF blend, respectively. Experimental data from FT-IR spectroscopy point to the formation of interfacial adhesion on the interface between PA11/PVDF blends and VA-MA during the blending and hot-pressing processes.

Figure 7. The FT-IR spectrum of (a) copolymer VA-MA and (b) PA11/PVDF (40:60) blends.

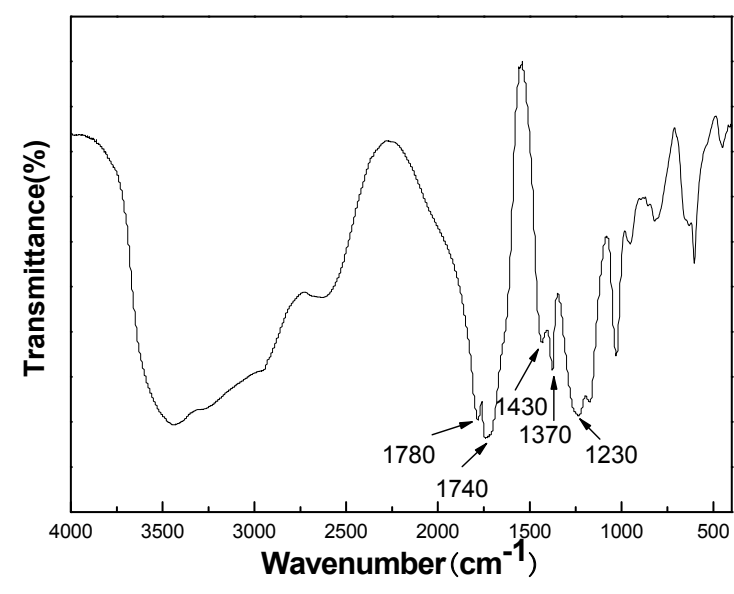

(a)

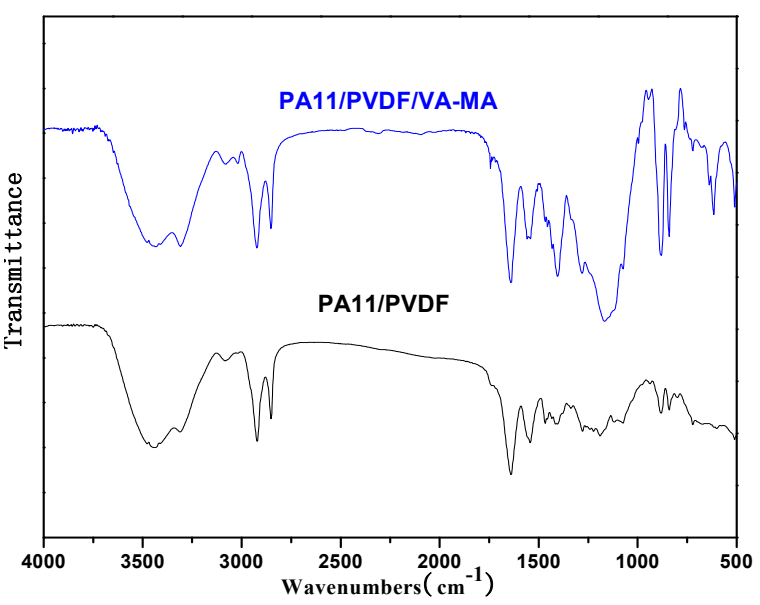

(b)

From Figure $7 \mathrm{~b}$ we can see that the absorption peak of wave number is $1780 \mathrm{~cm}^{-1}$ for carboxylic acid $\mathrm{C}=\mathrm{O}$ stretching vibration and $1230 \mathrm{~cm}^{-1}$ for $\mathrm{C}-\mathrm{O}$ stretching vibration; they are the characteristic structures of acid anhydride. The wave number is $1780 \mathrm{~cm}^{-1}$ for carboxyl ester $\mathrm{C}=\mathrm{O}$ stretching vibration and $1370 \mathrm{~cm}^{-1}$ for methyl groups $\mathrm{C}-\mathrm{H}$ flexural vibration. The intensity of absorption band in wave number $1370 \mathrm{~cm}^{-1}$ is greater than in $1430 \mathrm{~cm}^{-1}$, which is an obvious characteristic of vinyl acetate structure. So it is proved the malefic anhydride and vinyl acetate copolymerization took place.

In Figure 7a, the characteristic double peaks of the acid anhydride group at $1780 \mathrm{~cm}^{-1}$ and $1230 \mathrm{~cm}^{-1}$ disappear when VA-MA molecules graft with -NH on the surface of PA11 particles, and the peak at 
$1414 \mathrm{~cm}^{-1}$ indicates the formation of the cyclic amide group [22-25]. Also, the characteristic peaks of the ester carboxyl at $1740 \mathrm{~cm}^{-1}$ became weaker. This indicates that the copolymer VA-MA has been successfully introduced to the polymer main chain. These phenomena indicated that a new blend (PA11-g-VA-MA) was produced by the reaction, acting as the real compatilizer to promote the reaction between VA-MA and PA11. Therefore, adding a proper amount of VA-MA is an important measure to improve the electrical properties. The graft reaction of PA11 and VA-MA can be exhibited by reference [11]. As an effective compatilizer, PA11-g-VA-MA can make PA11 and VA-MA compatible. At the same time, PVDF is also compatible with VA-MA. The reason is that two carbonyls and one oxygen atom with pentavalent rings are coexistent in the VA-MA molecular chain, which can react with the a-phase hydrogen atom in PVDF. The hydrogen atom of VA-MA may also simultaneously interact with the electronegative fluoride atom in PVDF. Those intermolecular functions improve the compatibility between the PA11 and PVDF.

\section{Conclusions}

We have introduced a simple, efficient, and repeatable route to produce high-K blends composed of PA11 and PVDF. Through the measurements of dielectric constant K, dielectric loss tan $\delta$ and piezoelectric constant $\mathrm{d}_{33}$ after polarizing process at room temperature, it is shown that the PA11/PVDF blends with VA-MA are of higher dielectric constant $\mathrm{K}$ and lower dielectric loss tan $\delta$.

By analyzing the electric properties of the PA11/VA-MA/PVDF blends and inquiring into its intermolecular interface microstructures, this article probes into the typical layer-by-layer structure that appears on the interface of the intermolecular configuration. This may be attributed to the compatibleness between PA1land PVDF caused by the VA-MA, which makes the electron move among crystallization grains, and then improve the polarization and electric properties.

Compatibility plays an important role in polymeric blends. The dielectric stability and reliable mechanical stability make these all-polymer films excellent candidates for future high-K dielectrics such as the manufacture devices and electronics. This high-performance all-polymer blend is promising in terms of use as a flexible dielectric material. It provides an opportunity to fabricate more high functional applications and more innovative products.

From the intermolecular interface microstructures, the compatilizer VA-MA was applied to this blend system to react with both polymers (PA11 and PVDF) so as to improve the compatibility between PA11 and PVDF. The phase separation disappears on the interface intermolecular configuration. Therefore, the PA11/PVDF blends are of higher flexible property in testing mechanical properties. Compared with our previous research, the PA11/PVDF blend displayed a high dielectric constant, but the preparation process is complex with low yield and length time, which is difficult for application. The dielectric and piezoelectric properties of the PA11/PVDF with the compatilizer VA-MA were determined respectively according to the applications, such as micro-capacitor, sensor, and so on.

\section{Acknowledgments}

This work was supported by the Key Program for International Science and Technology Cooperation Projects of Shaanxi province (No.2012KW-09, 2014KW10-03), the Doctoral Program Foundation of Institutions of Higher Education of China Research Fund for the Doctoral Program of Higher Education 
of China (No. 20120205120010), and The Fundamental Research Funds for the Central Universities of Chang'an Universities (No. 0009-2014G1211012).

\section{Conflicts of Interest}

The authors declare no conflict of interest.

\section{References}

1. Zhang, Q.M.; Li, H.F.; Poh, M.; Xia, F.; Cheng, Z.Y.; Xu, H.S.; Huang, C. An all-organic composite actuator material with a high dielectric constant. Nature 2002, 419, 284-287.

2. Scheinbeim, J.I.; Newman, B.A.; Ma, Z.Y.; Lee, J.W. Electrostrictive respons of elastomeric polymers. Polym. Prepr. 1992, 33, 385-386.

3. Nishino, A. Capacitors: Operating principles, current market and technical trends. J. Power Sources 1996, 60, 137-147.

4. Schuster, B.E.; Wei, Q.; Hufnagel, T.C.; Ramesh, K.T. Size-independent strength and deformation mode in compression of a Pd-based metallic glass. Acta Mater. 2008, 56, 5091-5100.

5. Dang, Z.M.; Yan, W.T.; Xu, H.P. Novel high-dielectric-permittivity poly(vinylidene fluoride)/polypropylene blend composites: The influence of the poly(vinylidene fluoride) concentration and compatibilizer. J. Appl. Polym. Sci. 2007, 105, 3649-3655.

6. Arbatti, M.; Shan, X.B.; Chen, Z.Y. Ceramic-polymer composites with high dielectric constant. Adv. Mater. 2007, 19, 1369-1372.

7. Schneuwly, A.; Gröning, P.; Schlapbach, L.; Brüesch, P.; Carlen, M.W.; Gallay, R. Temperature-dependent dielectric breakdown strength of oil impregnated polypropylene foils. Mater. Sci. Eng. B 1998, 54, 182-188.

8. Bayer, I.S.; Biswas, A.; Szczech, J.B.; Suhir, E.; Norton, M.G. Radio frequency functional capacitors made of all-organic composites of thiourea in field-responsive polymers. Appl. Phys. Lett. 2008, 92, 083303:1-083303:3.

9. Newnham, R.E. Composites Electroceramics. Ferroelectrics 1986, 68, 1-32.

10. Dong, L.J.; Xiong, C.X.; Quan, H.Y.; Zhao, G.H. Polyvinyl-butyral/lead zirconate titanates composites with high dielectric constant and low dielectric loss. Scr. Mater. 2006, 55, 835-837.

11. Yao, J.L.; Xiong, C.X.; Dong, L.J.; Chen, C.; Lei, Y.A.; Li, R. Enhancement of dielectric constant and piezoelectric coefficient of ceramic-polymer composites by interface chelation. J. Mater. Chem. 2009, 19, 2817-2821.

12. Shen, Y.; Lin, Y.; Lin, M.; Nan, C.W. High dielectric performance of polymer composite films induced by a percolating interparticle barrier layer. Adv. Mater. 2007, 19, 1418-1422.

13. Bhattacharyya, D.; Yoon, W.J.; Berger, P.R.; Timmons, R.B. Plasma-polymerized multistacked organic bipolar films: A new approach to flexible high-k dielectrics. Adv. Mater. 2008, 20, 2383-2388.

14. Nair, S.S.; Ramesh, C.K.; Tashiro, K. Crystalline Phases in Nylon11: Studies Using HTWAXS and HTFTIR. Macromolecules 2006, 39, 2841-2848. 
15. Dang, Z.M.; Nan, C.W.; Xie, D.; Zhang, Y.H.; Tjong, S.C. Dielectric behavior and dependence of percolation threshold on the conductivity of fillers in semiconductor polymer composites. Appl. Phys. Lett. 2004, 85, 97-99.

16. Biswas, A.; Bayer, I.S.; Marken, B.; Pounds, T.D.; Norton, M.G. Networks of ultra-fine Ag nanocrystals in a Teflon $\mathrm{AF}^{\circledR}$ matrix by vapour phase e-beam-assisted deposition. Nanotechnology 2007, 18, 1-6.

17. Gao, Q.; Scheinbeim, J. Dipolar intermolecular interactions, structural development, and electromechanical properties in ferroelectric polymer blends of nylon11 and poly(vinylidene fluoride). Macromolecules 2000, 33, 7564-7572.

18. Hsien, S.; Woo, E.M. Weak interaction,marginal miscibility and ring band spherulites in blends of poly(vinylidene fluoride) with polyster. J. Appl. Polym. Sci. 2008, 107, 766-777.

19. Chiu, H.J. Spherulite morphology and crystallization kinetics of poly(vinylidene fluoride)/poly(vinyl acetate). J. Polym. Res. 2002, 9, 169-174.

20. Esterly, D.M.; Love, B.J. Phase transformation to poly(vinylidene fluoride) by milling. J. Polym. Sci. B Polym. Phys. 2004, 42, 91-97.

21. Li, Y.J.; Xu, M.; Feng, J.Q.; Cao, X.L.; Yu, Y.F.; Dang, Z.M. Effect of the matrix crystallinity on the percolation threshold and dielectric behavior in percolative composites. J. Appl. Polym. Sci. 2007, 106, 3359-3365.

22. Li, R.; Xiong, C.X.; Kuang, D.L.; Dong, L.J.; Lei, Y.A. Polyamide 11/poly(vinylidene fluoride) blends as novel blends Flexible Materials for Capacitors. Macromol. Rapid Commun. 2008, 29, 1449-1454.

23. Wang, T.; Liu, D.; Xiong, C.X. Synthesis of EVA-g-MAH and its compatibilization effect to PA11/PVC blends. J. Mater. Sci. 2007, 42, 3398-3407.

24. Newman, B.A.; Scheinbeim, J.I. The piezoelectricity of poly(vinylidene fluoride). Jpn. J. Appl. Phys. 1980, 51, 5161-5164.

25. Jing, Q.; Lu, X.Y. Experimental study on the stability levulinic acid in near critical water. Chem. React. Eng. Technol. 2007, 23, 474-476.

(C) 2014 by the authors; licensee MDPI, Basel, Switzerland. This article is an open access article distributed under the terms and conditions of the Creative Commons Attribution license (http://creativecommons.org/licenses/by/3.0/). 